\title{
Photographic evidence of luminescence during faulting in granite
}

\author{
Mamoru Kato ${ }^{1}$, Yuta Mitsui ${ }^{2}$, and Takashi Yanagidani ${ }^{3}$ \\ ${ }^{1}$ Graduate School of Human and Environmental Studies, Kyoto University, Kyoto 606-8501, Japan \\ ${ }^{2}$ Department of Geophysics, Kyoto University, Kyoto 606-8502, Japan \\ ${ }^{3}$ Disaster Prevention Research Institute, Kyoto University, Uji 611-0011, Japan
}

(Received December 15, 2008; Revised July 14, 2009; Accepted February 13, 2010; Online published July 9, 2010)

\begin{abstract}
Reports on the sky being brightly illuminated before, during, or after large earthquakes have existed for many centuries. Physical processes behind this phenomena, however, has not been fully explained despite large public interest in this phenomena. In this report, we present photographic evidence that visible light is emitted from faults. We observe luminescence that occurs simultaneously with the brittle faulting, and spatial distribution of the luminescence coincides the faulting pattern. This luminescence consists of several colors and is recordable with commercial digital cameras. In our experimental setting, it was observed only during fracture of granite, and we postulate that piezoelectricity of quartz in granite plays a major role in its occurrence.
\end{abstract}

Key words: Earthquake light, rock fracture, faulting.

\section{Introduction}

Some earthquakes produce lights and illumination of the sky before and during seismic rupture. This phenomenon, earthquake light, is a typical example of a macroscopic anomaly associated with earthquakes. Anecdotes of such luminous phenomenon are reported in ancient Egyptian, Chinese, and Japanese documents (Musha, 1931; Terada, 1931; Derr, 1973; Ikeya and Takaki, 1996). Photographic evidence of the earthquake light obtained during earthquake swarm activities in Matsushiro, Japan, in 1960s, caught the attention of geophysicists (Yasui, 1968, 1971; Derr, 1986). Similar sightings were reported in the M 7.2 1995 Hyogoken-nambu (Kobe) Earthquake (Tsukuda, 1997; Kamogawa et al., 2005), which heightened public interest in this phenomenon. Despite popular interest on this phenomenon, which could be an immediate earthquake precursor, scientific research on earthquake light has been limited. How such a luminous phenomenon occurs, and whether and how it relates to faulting processes is not well understood, but given that luminescence commonly appears during the fracture of brittle material, several promising hypotheses have been proposed in which earthquake light is linked either to pre-seismic stress accumulation or co-seismic faulting (Scholz, 1972; Lockner et al., 1983; Schloessin, 1985; Brady and Rowell, 1986; Derr, 1986; Freund, 2003).

Difficulty in studying earthquake light arises from the scarcity of scientific observations. Previous observations of earthquake related luminous phenomena were made by chance, and descriptions by witnesses are often sketchy, partly owing to the very short time constant of this phe-

Copyright (c) The Society of Geomagnetism and Earth, Planetary and Space Sciences (SGEPSS); The Seismological Society of Japan; The Volcanological Society of Japan; The Geodetic Society of Japan; The Japanese Society for Planetary Sciences; TERRAPUB.

doi:10.5047/eps.2010.02.004 nomena. A lack of repeated observation is also an inherent problem with this type of potential earthquake precursor. Our approach in this study is to model earthquake light in carefully controlled laboratory experiments. We fractured crustal rocks in the laboratory and observed luminescence and light during failure. We are especially interested in the spatial pattern of luminescence, and here we report photographic evidence of this light being radiated from faults formed in intact rock samples.

\section{Methods}

Our experiment uses the standard uniaxial loading system commonly employed in rock physics: a column-shaped rock specimen is sandwiched between two steel platens and the load is applied until the specimen undergoes the final brittle fracture. Fracture in the uniaxial test is always explosive, but when the apparatus and specimen are carefully prepared, such explosive fracture accompanies faulting planes in the specimen (Yanagidani et al., 1985), which is the key technical aspect in our study. Typical size of our specimen is $5 \mathrm{~cm}$ in diameter and $12 \mathrm{~cm}$ in height. Our experiments are conducted in the ambient atmosphere at room temperature and humidity.

Our focus is on the spatial pattern of luminescence that has appeared to be overlooked in previous experimental studies. We photographically record the luminescence with digital single-lens reflex cameras. A common approach in studying luminous phenomena is spectroscopy to infer the excitation mechanism. Luminescence with faulting involves a very short time constant, and spectroscopy of short, weak luminescence always requires signal-enhancing devices. Placing such devices in the apparatus would limit the field of vision and make simultaneous observation of spectrum and spatial pattern difficult. Similarly, we do not use strain measuring devices, as these could become obstacles in photography and might alter the electric field at 
(a)

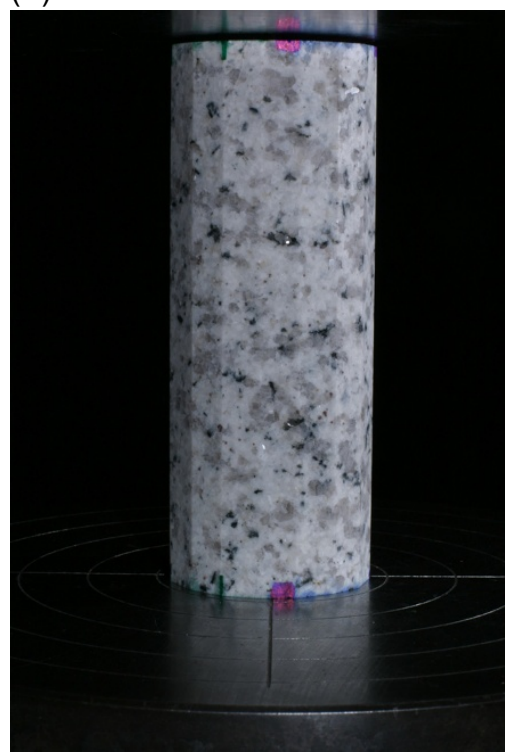

(d)

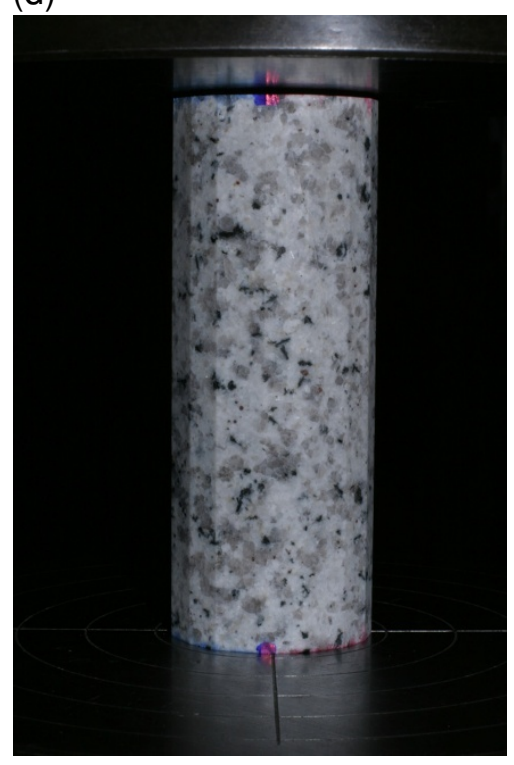

(b)

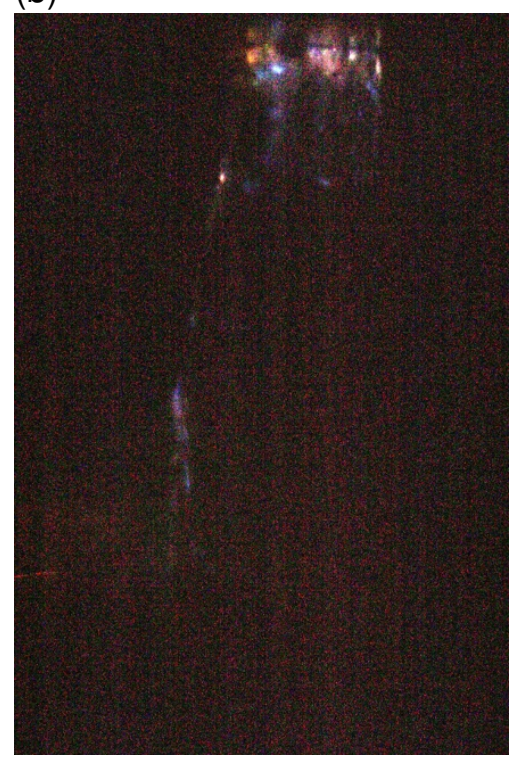

(e)

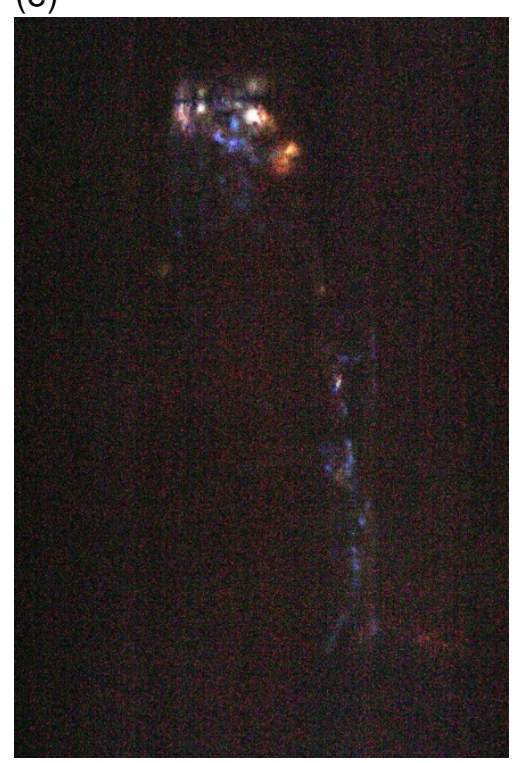

(c)

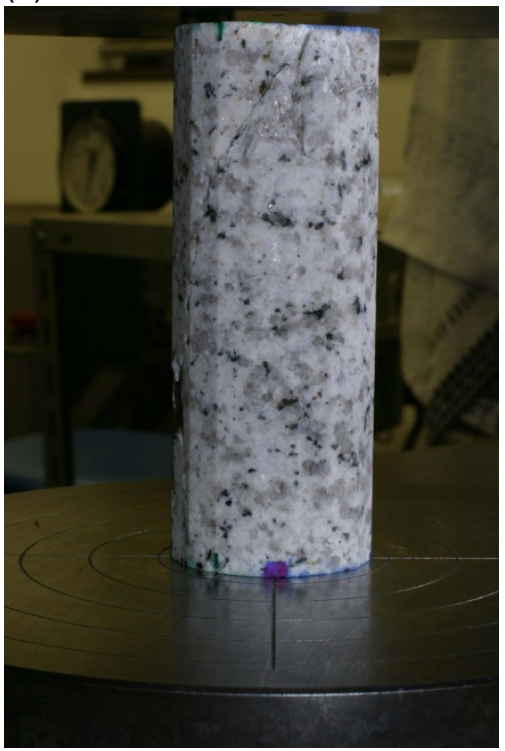

(f)

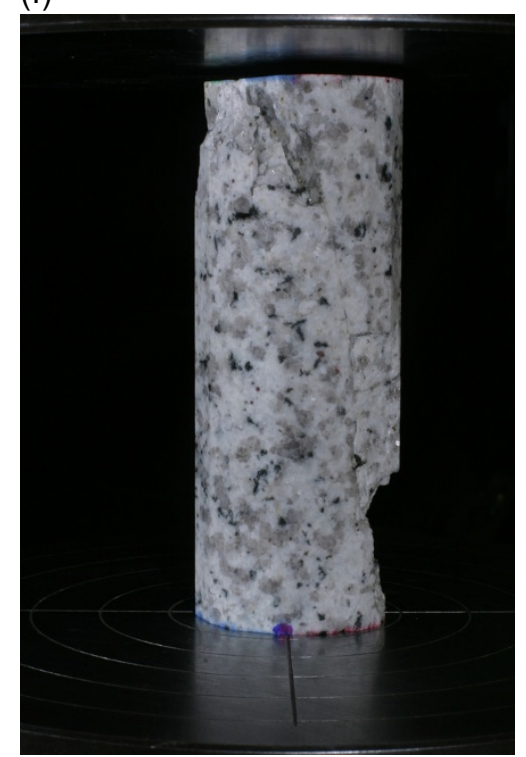

Fig. 1. Photographic images for run I4-1. The specimen is a coarse grained Inada granite. With sensitivity of ISO 3200 and F (lens opening) of 1.4, our photographs are similar to images that humans visually sense. Shutter speed is set to $3 \mathrm{~s}$ and photographs are saved in uncompressed data format. Images in the second row (d-f) are taken from the direction $90^{\circ}$ counterclockwise from the angle for the first row images (a-c) for the same run. (a) and (d): images of the rock specimen before the loading, which are taken during daylight. Color markers on both ends of the sample are drawn to record the sample orientation and are irrelevant to the result. Size of the specimen is $5 \mathrm{~cm}$ in diameter and $12 \mathrm{~cm}$ in height. (b) and (e): images of luminescence radiated from this specimen. These images are intensified to highlight locations of the bright spots by simply multiplying each of RGB value 4 folds. (c) and (f): Images of specimen after the faulting. For this experimental run, we successfully recovered two largest fragments of this specimen that are split by a well developed fault plane spontaneously developed during its fracture and reassemble them to reconstruct the original column shape. Two largest fragments are assembled together to the original column shape. The fault plane runs from the top center downward to the left in (c), and from the top left to the bottom right in (f).

the surface of the specimen. We accordingly concentrate on the spatial characteristics of the luminescence and make conjectures spectral characteristics from the colors on photographs.

The Canon EOS10D, a commercial product digital camera, is our sensor and has sufficient sensitivity for our purpose. Multiple (two to four) cameras are placed at a distance of $0.5 \mathrm{~m}$ from the rock specimen to record the process simultaneously from different angles. Successful photography of luminescence is possible only when the entire apparatus and cameras are placed in the darkroom condition.
Yet it is much brighter than the background and instrumental noises, and our photographs are unambiguous records of the luminescence. The shutter is open for $3 \mathrm{~s}$ at each frame, with short pauses in between which are required to save image data. Continuous data (image) acquisition starts at several seconds before the initial loading and ends at several seconds after the faulting. The loading rate is of order of $10^{4} \mathrm{~Pa} \mathrm{~s}^{-1}$, several orders higher than the tectonic stress loading in the Earth. The final faulting occurs within $30 \mathrm{~min}$ after the loading is initiated in all experimental runs, and luminescence occurs at the faulting. In each experimental 
(a)

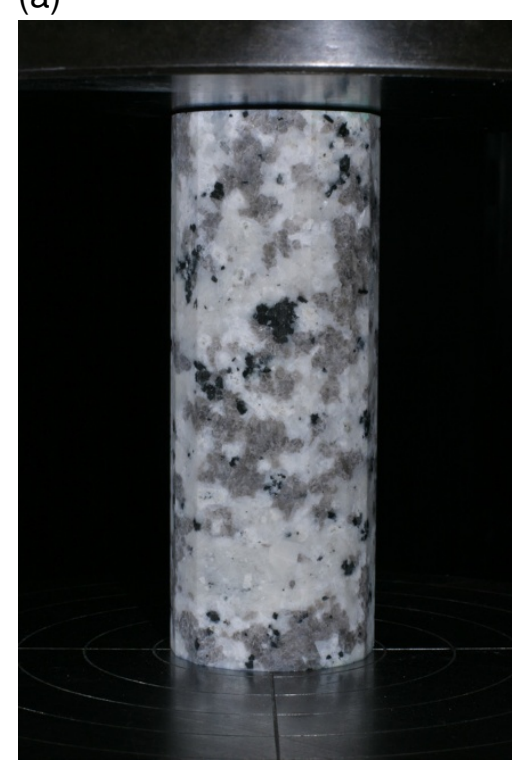

(c)

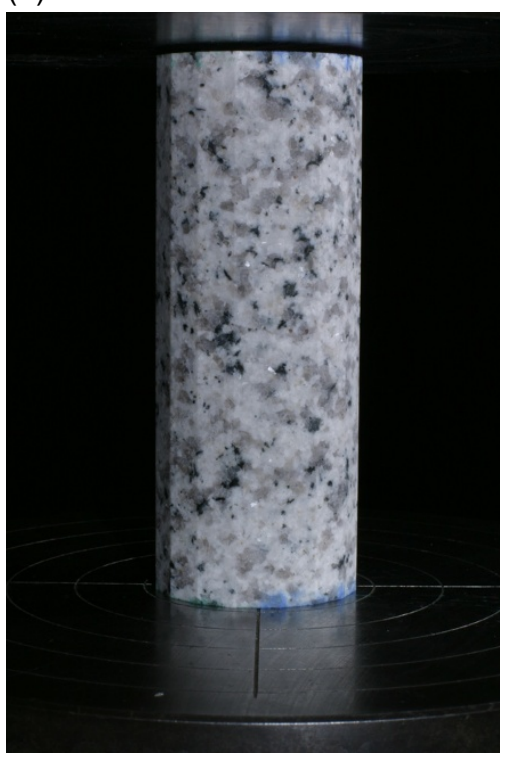

(b)

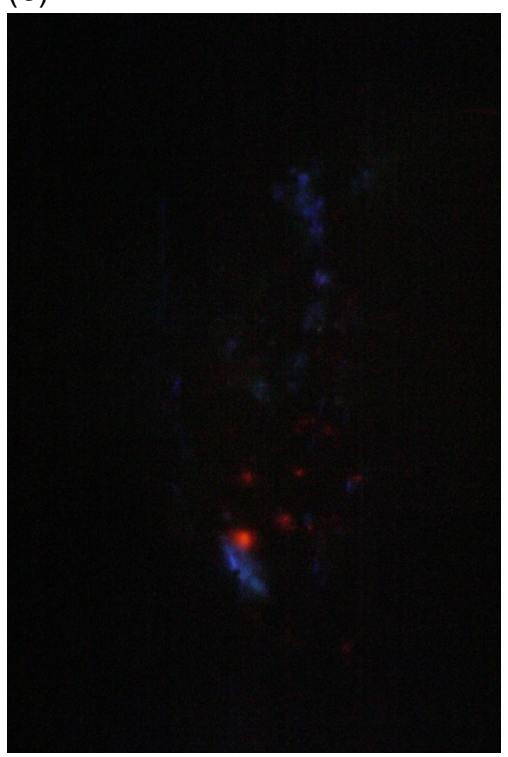

(d)

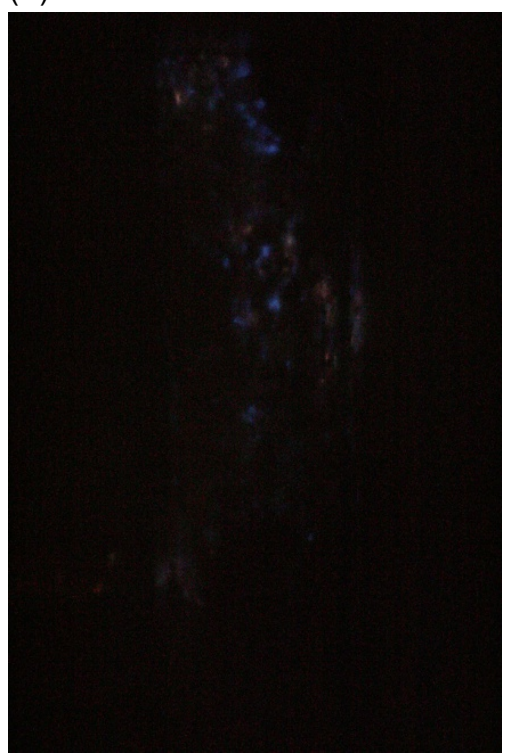

Fig. 2. Comparison of luminescence with two different granites, Fujioka (a and b, run F4-2) and Inada (c and d, run I3-1). Note that Fujioka granite is coarser grained granite. (a) and (c): Images of the specimen before the loading. Size of specimens are $5 \mathrm{~cm}$ in diameter and $12 \mathrm{~cm}$ in height. (b) and (d): Images of luminescence during their brittle fracture, taken from the same position as the (a) and (c), respectively. Images are not intensified. Bright red and blue-violet spots are identified on the lower half of the specimen for Fujioka granite, while weak blue-violet spots are identified on the lower half of the specimen for Inada granite.

run, we utilize images of the specimen taken both before the loading and after the faulting under daylight from the same position to locate the luminescence afterward.

Brightness of the luminescence depends on the rock types. Some rock types, such as basalt, marble, and quartzpoor tornalite do not exhibit luminescence in our experiments. We observe the strongest luminescence with granite, the most common igneous rock in the Earth's crust, and hereafter we focus on granite in this report. Luminescence occurs always simultaneously with the faulting of the specimen, i.e., coseismic. In all successful recordings, no luminescence is located at a distance from the specimen, and this process takes place very close to the specimen, or on the specimen. Luminescence is observed only for dry samples, but never for wet samples, the latter being submerged in distilled water for several days before experiments while dry samples are kept in the desiccator.

\section{Results and Discussion}

We found that the faulting plane in the rock specimen is often the place of bright luminescence. Bright luminescence typically appears as a collection of bright spots. In Fig. 1, which is a typical image of bright luminescence for a coarse grained granite specimen, illuminated bright spots align diagonally from the upper left corner to the lower right corner on the specimen (Fig. 1(e)). This alignment is on the fault plane that is spontaneously developed during its fracture (Fig. 1(e, f)), which demonstrates that bright luminescence occurs on the fault plane.

Two colors, blue-violet and orange, are dominant in our 


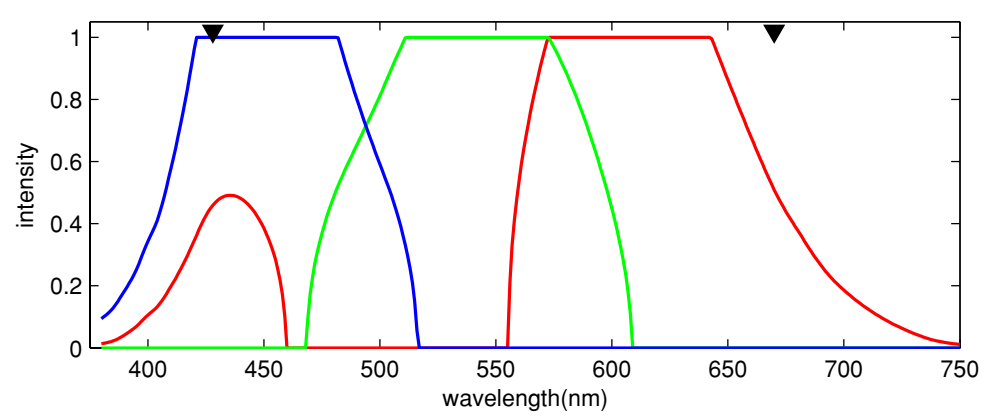

Fig. 3. Approximate RGB values for visible wavelength. Two inverted triangles indicate wavelength for $\mathrm{N}_{2}^{+} 1 \mathrm{~N}$ and $\mathrm{N}_{2} 1 \mathrm{P}$ nitrogen emission, respectively.

photographs (Fig. 1). These colors coexist on one image, and blue-violet is usually identified with a brighter luminescence. Brightness of the luminescence is apparently controlled by the texture of the rock specimen. We repeatedly observe that coarse-grained granites produce brighter luminescence than fine-grained granites (Fig. 2). For coarsegrained specimens, we are able to compare texture of the specimen and the spatial distribution of luminescence. Location of bright blue-violet spots are spatially correlated with quartz grains; they are located adjacent to quartz grains but not on quartz themselves. Both the occurrence and colors of the luminescence are reproducible with the same type of rock specimen.

We postulate that bright illuminated spots are likely nitrogen molecules excited by an electric discharge or spark at faulting. Before the faulting, piezoelectric quartz minerals in the specimen under stress produces a piezoelectric field, which is neutralized by associated bound charges (Yoshida et al., 1997). At the faulting, the direct piezoelectric field disappears immediately, but bound charges would be slow to dissolve, which causes an electrostatic discharge, such as the creeping discharge in the close vicinity of the specimen. Control by the grain sizes on brightness of the luminescence would be consequence of the texture itself and strain concentration in the specimen. Strain is localized in the area of the future faulting plane (Yanagidani et al., 1985). Large strain would induce a large piezoelectric effect, especially when large grains are present, and an alignment of bright spots at the rupture plane is therefore a consequence of highly strained zones in the specimen. Rough surface created at the fault plane is also favorable in inducing electric discharge or spark. The absence of luminescence with wet samples is explained by this while piezoelectricity of quartz works even in the wet specimen, as water being a good conductor would enable charges to move away and inhibit charge buildup.

One of the brightest blue-violet and orange spots observed in our experiments are those that appear in the lower half of Fig. 2(b). The sRGB values for these spots are approximately $0.64 / 0.03 / 0.03 /$ and $0.30 / 0.04 / 0.45$. Let us note that in this report we quote linear sRGB values, which are values converted from standard nonlinear sRGB by removing the gamma correction for $\gamma=2$.4. The observed sRGB values do not match exactly the values for nitrogen emission wavelengths, $670 \mathrm{~nm}$ for $\mathrm{N}_{2} 1 \mathrm{P}$ (approximately $0.50 / 0 / 0$ ) and $428 \mathrm{~nm}$ for $\mathrm{N}_{2}^{+} 1 \mathrm{~N}$ (approximately $0.45 / 0.00 / 1.00$ ), re-

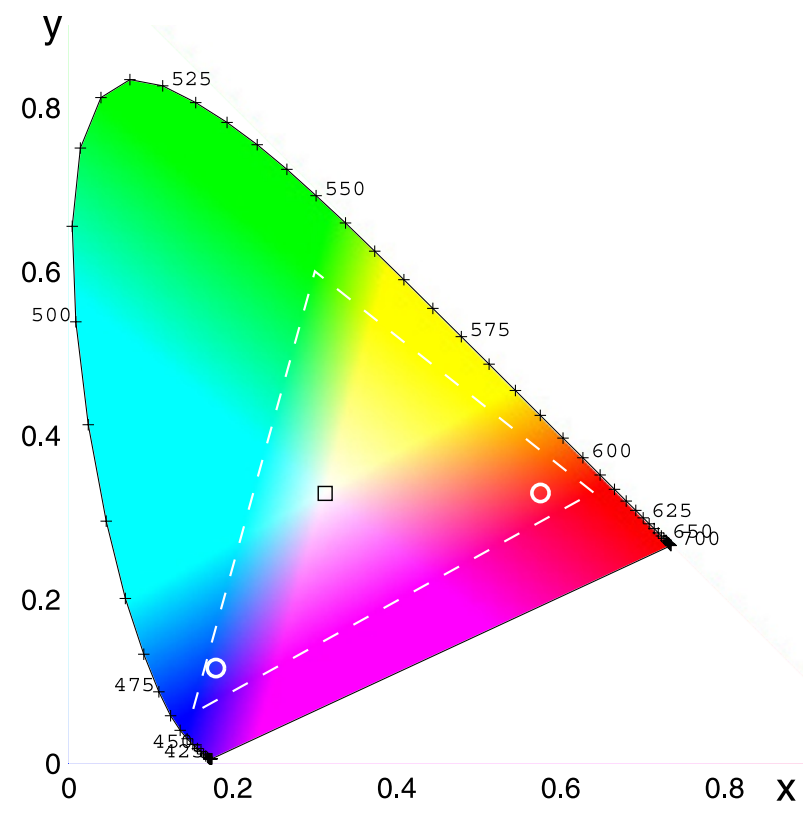

Fig. 4. CIE xy chromaticity diagram showing sRGB color space and locations of primaries. The open square shown in the center is the D65 white point. The RGB values in this diagram are scaled by luminnance $\mathrm{Y}$ so that D65 corresponds to RGB values of 1.0/1.0/1.0. The outer curved boundary is the monochromatic locus, with wavelengths are shown in nanometers and + are plotted at $5 \mathrm{~nm}$ interval. Apices of the dashed triangles are R, G and B primaries for sRGB. Two open circles respectively indicate observed sRGB values for two bright spots. Note that these circles are apparently located on the line connecting the D65 white points which has equal RGB values, and R and B primaries, respectively.

spectively (Fig. 3). One reason for this discrepancy is the short time constant of luminescence. Weak signals tend to be recorded by our devices as gray, and all these RGB values are equal. Short signal is a form of weak signal, and such an effect also would distort values for these bright spots slightly (Fig. 4). The other possibility is contamination from emission at other wavelengths. Excitation of nitrogen often occurs simultaneously at several wavelengths, such as that observed with the sprites in the stratopause (e.g., Heavner et al., 2000). We also confirmed that our devices are sensitive to infrared (as long as $953 \mathrm{~nm}$ ) and ultraviolet lights (as short as $365 \mathrm{~nm}$ ), and emission from nitrogen outside the visible range could affect the recorded values as well. We are not able to decompose the color of the photographic images into wavelengths uniquely, and 
further spectral analysis is desirable to confirm the details of luminescence that emits at least two visible colors.

Our results are not easily reconcilable with the hypothesis of Brady and Rowell (1986), that is, charged particle bombardment emitted at fracture, or fractoemission, excite the nitrogen molecules in the surrounding atmosphere. Several differences exist between our and their observations. Brady and Rowell (1986) used solely fine-grained samples which emit weak or no luminescence in our experiments. Our luminescence is bright enough to be seen by the naked human eye as a short-lived flash and is located only in the close vicinity of the specimen. Enhanced fractoemission in the wet condition (Enomoto and Hashimoto, 1990) also contradicts our results. One possibility is that luminescence with rock fracture is a compound process of these two processes: bombardment processes are dominant in the low stress regime and responsible for weak background luminescence, while piezoelectricity works in a high-stress regime, causing bright spots. Here we naively use the term low- and high-stress regimes, where we loosely define the high-stress regime as the condition such that significant piezoelectric effect is induced, the details of which depend of the characteristics of specimen and the experimental environment. Faulting of intact rock samples in uniaxial condition occurs only when the specimen is subject to a high stress as a result of the precise alignment of apparatus and specimen, and large piezoelectricy is expected in such a condition. Our camera was not sensitive enough to record what Brady and Rowell recorded or, similarly, what Martelli et al. (1989) and Kawaguchi (1998) observed, so that we are not able to rule out that the weaker luminescence does not occur in our experimental condition. At the same time, their devices were not able to captivate variation in brightness, and our observation of bright spots on the fault is a new finding.

\section{Concluding Remarks}

The luminescence we observed in the laboratory is a purely coseismic process and the time constant is very short. The light is emitted only during the rock rupture, and we observe neither light emission during the loading (preseismic) nor long-lasting light. Some sighting reports state that duration of earthquake light is more than minutes (Derr, 1973, 1986; St-Laurent, 2000; Kamogawa et al., 2005), several orders longer than our observations. Even when we take the scaling with respect to the fault size into consideration, direct application of our observation to explain such sighting might not be straightforward. Our observation could be related to the fast moving luminous phenomena along the earthquake fault observed in Kobe (Kamogawa et $a l ., 2005)$. Further exploration of links between the laboratory experiments and the natural phenomena would be useful in understanding electromagnetic aspects of earthquake faulting processes.
Acknowledgments. This work is supported partly by a Grant in Aid for Exploratory Research by the Ministry of Education, Culture, Sports, Science and Technology (MEXT), Japan (T.Y., M.K.). We are grateful for discussion with Katsuya Kaneko, Yasuyuki Kano, and Osamu Tamada. Masashi Kamogawa and Chris Marone provided thoughtful comments on the earlier version.

\section{References}

Brady, B. T. and G. A. Rowell, Laboratory investigation of the electrodynamics of rock fracture, Nature, 321, 488-492, 1986.

Derr, J. S., Earthquake lights-A review of observations and present theories, Bull. Seismol. Soc. Am., 63, 2177-2187, 1973.

Derr, J. S., Luminous phenomena and their relationship to rock fracture, Nature, 321, 470, 1986.

Enomoto, Y. and Y. Hashimoto, Emission of charged particles from indentation fracture of rocks, Nature, 346, 641-643, 1990.

Freund, F. T., Rocks that crackle and sparkle and glow: strange preearthquake phenomena, J. Sci. Explor., 17, 37-71, 2003.

Heavner, M. J., D. D. Sentman, D. R. Moudry, E. M. Wescott, C. L. Siefring, J. S. Morrill, and E. J. Bucsela, Sprites, blue jets, and elves: Optical evidence of energy transport across the stratopause, in Atmospheric Science Across the Stratopause, edited by D. Siskind, AGU, Washington, 2000.

Ikeya, M. and S. Takaki, Electromagnetic fault for earthquake lightning, Jpn. J. Appl. Phys. Part 2, 35, 355-357, 1996.

Kamogawa, M., H. Ofuruton, and Y. Ohtsuki, Earthquake light: 1995 Kobe earthquake in Japan, Atmos. Res., 76, 438-444, 2005.

Kawaguchi, Y., Charged particle emission and luminescence upon bending fracture of granite, Jpn. J. Appl. Phys., 37, 3495-3499, 1998.

Lockner, D. A., M. J. S. Johnston, and J. D. Byerlee, A mechanism to explain the generation of earthquake lights, Nature, 302, 28-33, 1983.

Martelli, G., P. N. Smith, and A. J. Woodward, Light, radiofrequency emission and ionization effects associated with rock fracture, Geophys. J. Int., 98, 397-401, 1989.

Musha, K., On the luminous phenomenon that attended the Izu earthquake, November 26th, 1930, Bull. Earthq. Res. Inst. Univ. Tokyo, 9, 214-215, 1931.

Schloessin, H. H., Experiments on the electrification and luminescence of minerals and possible origins of EQLs and sferics, Ann. Geophys., 3, 709-720, 1985.

Scholz, C. H., Static fatigue of quartz, J. Geophys. Res., 77, 2104- 2114, 1972.

St-Laurent, F., The Saguenay, Quebec, Earthquake lights of November 1988-January 1989, Seismol. Res. Lett., 71, 160-174, 2000.

Terada, T., On luminous phenomena accompanying earthquakes, Bull. Earthq. Res. Inst. Univ. Tokyo, 9, 225-255, 1931.

Tsukuda, T., Size and some features of luminous sources associated with the 1995 Hyogo-ken Nanbu earthquake, J. Phys. Earth, 45, 73-82, 1997.

Yanagidani, T., S. Ehara, O. Nishizawa, K. Kusunose, and M. Terada, Localization of dilatancy in Ohshima granite under constant uniaxial stress, J. Geophys. Res., 90, 6840-6858, 1985.

Yasui, Y., A study on the luminous phenomena accompanied with earthquakes (part 1), Mem. Kakioka Mag. Obs., 13, 25-61, 1968.

Yasui, Y., A study on the luminous phenomena accompanied with earthquake (part 2), Mem. Kakioka Mag. Obs., 14, 67-77, 1971.

Yoshida, S., M. Uyeshima, and M. Nakatani, Electric potential changes associated with slip failure of granite: Preseismic and coseismic signals, J. Geophys. Res., 102, 14,883-14,897, 1997.

M. Kato (e-mail: mkato@gaia.h.kyoto-u.ac.jp), Y. Mitsui, and T. Yanagidani 